\title{
Intercostal Nerve Transfers to the Musculocutaneous-A Reliable Nerve Transfer for Restoration of Elbow Flexion in Birth-Related Brachial Plexus Injuries
}

\author{
1Orthopaedic Muset Institute, Barcelona, Spain \\ 2Department of Brachial Plexus Surgery, Deenanath Mangeshkar \\ Hospital, Erandwane, Opposite Mehendale Garage, Pune, India \\ ${ }^{3}$ Department of Hand and Upper Limb Surgery, Hospital Real San \\ Jose, Universidad Autónoma De Guadalajara and Universidad de \\ Coahuila Torreón, Mexico \\ ${ }^{4}$ Department of Orthopaedic, Hand and Microsurgery, Assiut \\ University, Assiut, Egypt
}

Alex Muset Lara ${ }^{1} \quad$ Anil Bhatia ${ }^{2} \quad$ Jorge Clifton Correa ${ }^{3}$ Tarek Abdalla El Gammal ${ }^{4}$

\begin{abstract}
Address for correspondence Anil Bhatia, MS (ortho), Deenanath Mangeshkar Hospital, Erandwane, Opposite Mehendale Garage, Pune 411004, India (e-mail: agbhatia63@gmail.com).
\end{abstract}

Indian J Plast Surg:2020;53:254-259

\begin{abstract}
Introduction There is consensus on the need for early microsurgical reconstruction in birth palsies involving three or more roots, that is, extensive partial palsies and total palsies. The fundamental principles of these operations are complete exploration and judicious use of the ruptured stumps by nerve grafting to suitable distal targets. The frequent observation of root avulsions in such cases makes it imperative to look for extraplexual nerve donors for some functions. Intercostal nerves are readily available in such patients.

Materials and Methods This is a study of 50 patients of extensive partial and total birth palsies operated upon by the senior author between 1995 and 2010. These included 33 patients with total palsies, 16 patients with near total palsies, and one patient with $\mathrm{C} 56$ deficit (operated upon more than 20 years ago). These children were all operated upon between 3 and 6 months of age, except for two patients in whom surgery was delayed till a year due to the phrenic nerve deficit noted at birth. Four intercostal nerves were transferred to the musculocutaneous nerve (MCN) by direct approximation with fibrin glue.

Results No respiratory complication was noted from the intercostal harvest. The follow-up ranged from 8 to 20 years (mean 10 years). As many as 48 of the 50 patients regained fully independent elbow flexion. In two cases, the procedure failed com-

Keywords

- birth palsies

- elbow flexion

- intercostal nerve transfers pletely and had to be salvaged with a free functioning muscle transfer and reuse of the intercostal nerves.

Conclusion Intercostal nerve transfers can be relied upon for restoration of elbow flexion in birth palsies. The ruptured roots can then be utilized for augmenting shoulder function in partial palsies or for hand function in total palsies.
\end{abstract}

\section{Introduction}

The role of primary nerve reconstruction in extensive partial and total birth-related brachial plexus palsies cannot be disputed. The objectives of nerve reconstruction have expanded with increasing experience in the use of nerve transfer techniques. The early efforts had been limited to harnessing available (ruptured) root stumps in the neck to reinnervate the paralyzed muscles. Addition of nerve transfers has given us the liberty to direct the growing axons from the roots to the shoulder (for the partial palsies) and the hand 
in cases of total palsies. The utility of intercostal nerves for neurotization has been known for a long time. Large series of posttraumatic palsies published in $1989\left(\right.$ Nagano $\left.^{1}\right)$ and 1991 (Chuang ${ }^{2}$ ) confirmed the consistent results for transfers to the musculocutaneous nerve (MCN). It is known that nerve repairs provide better results in younger patients. So, the outcomes in birth palsies should be predictable. Kawabata ${ }^{1}$ described their use in birth palsies in 2001. The comorbidity of associated rib fractures and scarring is not seen in these patients. Refinements in the harvest technique have helped avoid deformities of the chest wall with growth.

The authors present their experience in the use of intercostal nerves for restoration of elbow flexion in the management of birth-related brachial plexus injuries.

\section{Materials and Methods}

The authors studied a series of 50 patients operated upon till 2010. Of these, 33 patients were afflicted with total palsy (affection of all five roots of the brachial plexus), while 16 patients had presented with extensive partial palsies and weak hand function. Only one patient (operated upon more than 20 years ago) had had a C56 deficit with avulsions of both roots. At that time, there was no experience with the use of ulnar nerve transfers, and intercostal nerves were utilized for the biceps. In the extensive partial palsies, the existing hand function precluded transfers from the ulnar/median nerves, and the available root stumps had to be directed toward the shoulder and triceps.

As many as 48 patients were operated upon between 3 to 6 months of age. In two cases of total palsies, intercostal nerve transfers were delayed due to the associated phrenic nerve deficit. Nerve reconstruction for shoulder abduction and hand functions was performed primarily. Respiratory function improved by 9 months, and the operation for the biceps was performed separately at a year.

The transfers formed a part of a comprehensive strategy to restore shoulder and elbow functions in partial palsies and shoulder, elbow and and hand functions in total palsies. In each case, the supraclavicular brachial plexus was explored via an incision along the sternomastoid and along the clavicle. The phrenic nerve was isolated, and the roots were examined at the foramina medial to the phrenic nerve. The plexus was examined completely, and each affected root was traced to the foramen. The number of roots affected (in partial palsies) and the condition of the lower trunk in total palsies were noted.

The fundamental step in the operation was excision of the scarred segments of the roots and trunks, and nerve grafting from viable proximal stumps to suitable distal targets. Hence, the clinical situation at birth was recreated before reconstruction. Complete absence or poor hand function at 3 months automatically pointed to a severe lesion of the lower trunk. Spontaneous recovery in such cases has never been satisfactory in our experience. Hence, provision of reliable innervation of the hand is imperative, that is, sectioning the roots at the foramina and connecting the best available roots to these, using adequate cables of nerve grafts.
The spinal accessory nerve could be easily identified along its course over the deep surface of the trapezius muscle. It was traced as distally as possible to preserve the branches to the upper part of the muscle, divided and transferred by direct approximation to the suprascapular nerve. This nerve was divided at its origin from the upper trunk and trimmed till good fascicles were noted in the cross-section. Innervation of the rotator cuff was achieved in this manner, and this nerve transfer was an integral part of the procedure in each of these patients.

\section{Technique}

The intercostal transfer was performed using a separate thoracobrachial incision staggered in the axilla. In the beginning, the chest incision was separated from the exposure of the MCN with an intact skin bridge at the anterior axillary fold. Over the years, we have abandoned this practice and make one long incision. We have found that this reduces the time of surgery without any serious disadvantages. The MCN was exposed between the heads of the biceps, traced proximally and divided at its origin from the lateral cord. The incision was then extended medially along the sixth rib. The slips of the serratus anterior and the intercostal muscles were detached from the ribs (along the lower borders). Care was taken to avoid stripping the periosteum, as that would affect the shape of the chest wall with growth. The nerves were approached by splitting the intercostal muscles. At each level, the nerve was isolated anteriorly at or just medial to the costochondral junction, divided and, then, separated rapidly from the pleura, dividing the tiny branches into the intercostal muscles. An attempt was made to preserve the sensory branches to the skin of the chest (particularly in girls to retain sensation over the breast area), but mobilization of the nerves for direct approximation to the musculocutaneous nerve was given priority. Four intercostal nerves were harvested in each case (usually the $3 \mathrm{rd}$ to the 6th). They were approximated directly to the MCN using fibrin glue. The nerves were passed deep to the skin bridge in order to reach the target nerve in the cases where we made separate incisions.

The arm was held in adduction across the chest for a month along with a plaster support, extending around the head to prevent excess movements of the neck which could disturb the grafts placed there.

Subsequently, the arm was mobilized to restore motion at the elbow, wrist, fingers and thumb, while shoulder mobilization was restricted initially to avoid excessive stretching at the repair of the intercostals.

The children were then observed for gradual return of function.

\section{Results}

Most of the children (49/50) did not suffer any respiratory disturbance in the period following the harvest of the intercostal nerves. In one case of total palsy, utilization of the only available root stump necessitated separation of the phrenic nerve, which produced respiratory distress due to 
the paralysis of the diaphragm. The child needed 2 weeks of assisted ventilation and a further period of intensive care for 2 weeks.

Initial contractions of the biceps were noted at 6 months from the operation. During the first year, muscular contractions at biceps and brachialis muscles were automatic and inseparable from the normal function of the intercostals. Elbow flexion was observed when the patient coughs or breathed deeply. Functional dissociation from breathing and independent elbow flexion were achieved after 1 year from the operation.

In the initial period, the outcomes were measured using the Gilbert and Tassin scale. At 10 years follow-up, the biceps were graded using the Medical research Council (MRC) scale.

Thus, at 5 years, M3 score was noted in 48 of the 50 patients. At 10 years, all these patients had achieved M4 grade of strength in the biceps. (Illustrative case of extended partial palsy treated with nerve transfers of spinal accessory to suprascapular and four intercostal nerves to the musculocutaneous nerve [-Figs. 1-3]. Function regained at 4 years of age is shown).

In two cases, the biceps did not recover at all. We could not determine the cause of these failures. This was salvaged by subsequent use of a free microvascular gracilis transfer, with utilization of the same intercostal nerves once again.

The passive range of elbow flexion was fully preserved in each patient. All the patients could take their affected hands to their mouths in the normal manner. There was some

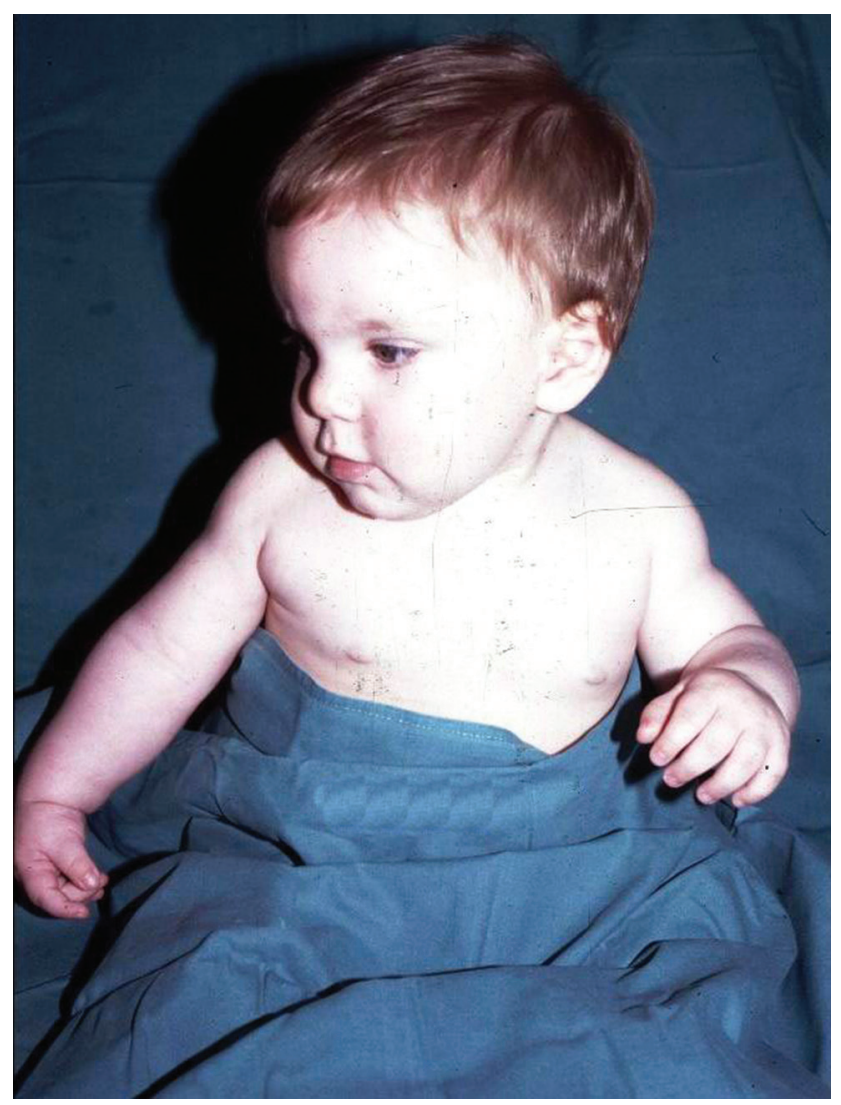

Fig. 1 Case of right extensive partial birth palsy at 4 months of age.

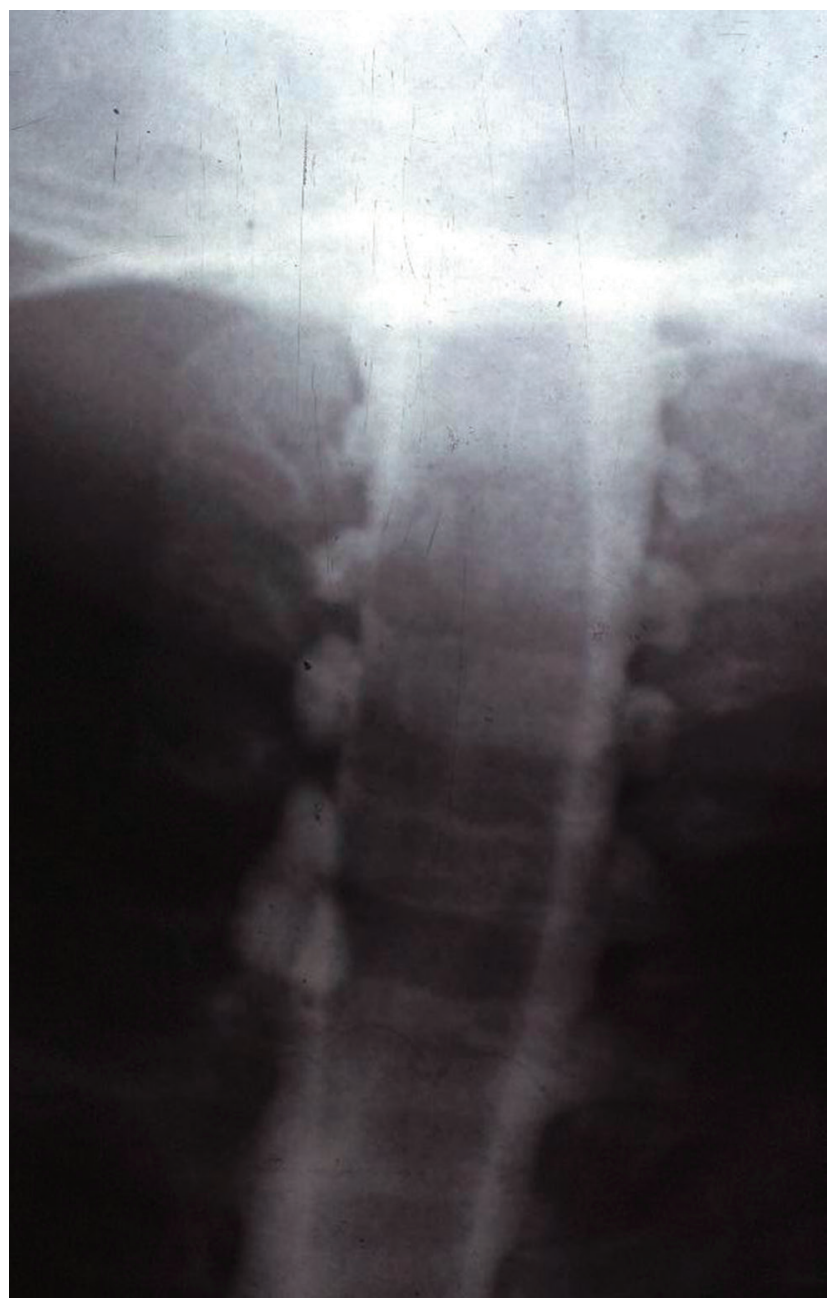

Fig. 2 Myelogram showing pseudomeningoceles, indicating avulsions of the right $\mathrm{C} 567$ roots.

residual weakness in the triceps with a maximal lag in extension of 15 degrees.

None of the patients reported chronic respiratory symptoms of expectoration nor repeated episodes of infection. In four cases, the family noticed grunting during sleep. In one of the patients who underwent plication of the diaphragm, dyspnea during exercise was noted. Repeated episodes of acute bronchospasm with wheezing was reported in four patients. The patients were treated satisfactorily in the outpatients' clinic.

Asymmetry in the two sides of the chest was noted in the $\mathrm{X}$-ray of the patient, with paralysis of the diaphragm.

At rest, each patient maintained normal respiratory rate (20/minute), pulse (64-129 beats/minute), and normal oxygen saturation in the blood (97-100\%) while breathing room air.

Pulmonary function tests on spirometry, too, consistently revealed normal values for each patient at long term follow-up.

\section{Discussion}

Early nerve reconstruction in extensive birth-related brachial plexus injuries does not require justification. In fact, 

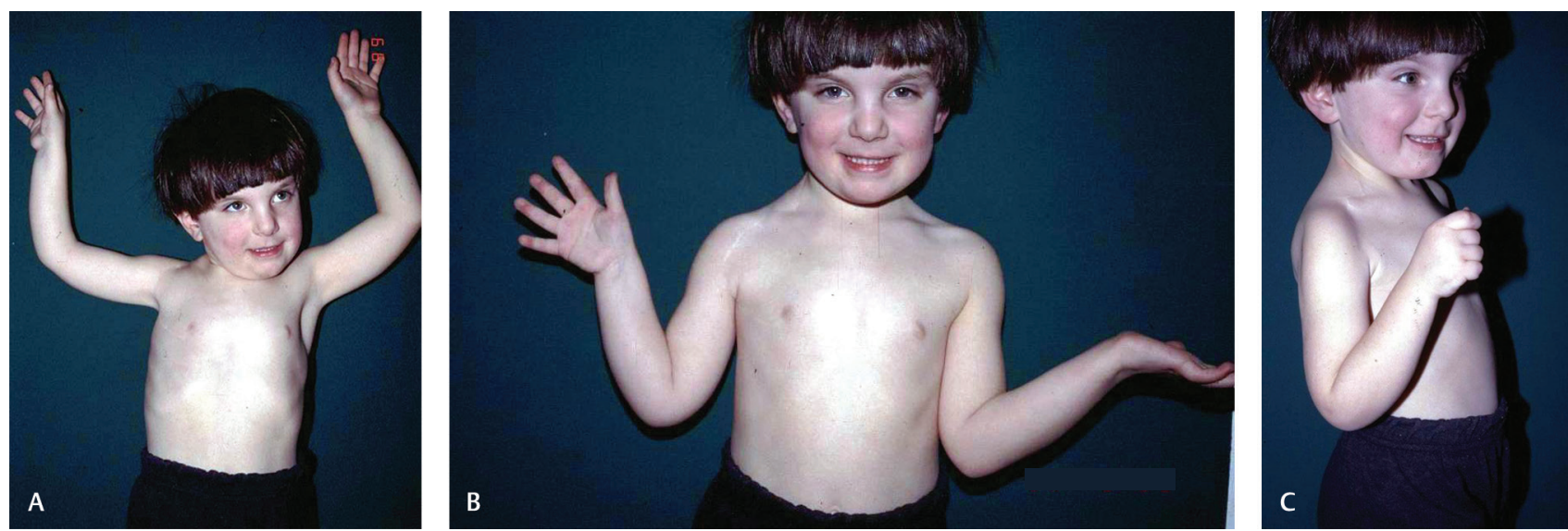

Fig. 3 Spinal accessory to suprascapular and intercostals to musculocutaneous nerve transfers done. Result at 4 years. (a). Abduction; (b). External rotation with arm adducted; (c). Elbow flexion.

with increasing confidence in the results of nerve repairs, current strategies aim to maximize functions in the paralyzed upper limb rather than mere creation of a helping hand. Comprehensive exploration of the injured plexus, excision of the scared segments of the roots and trunks, and nerve grafting from utilizable (ruptured roots) to suitable distal targets are fundamental steps of the nerve operation. In partial palsies where the lower trunk is spared, the upper and middle trunks are targeted. In total palsies, there is consensus that the lower trunk gets priority. Such injuries invariably include avulsions at one or more levels, and this decreases the available sources of innervation. Hence, the positive experience with extraplexual nerve transfers (spinal accessory and intercostal nerves) is invaluable in formulating a strategy that addresses the deficit at the shoulder, elbow and the hand.

In extensive partial palsies with partial involvement of the lower trunk, the weak hand function precludes use of fascicles of the ulnar and/or median nerves for elbow flexion. In addition, one often finds avulsions of the roots at multiple levels. Surgery is indicated as the shoulder and elbow functions are unlikely to improve if they are still absent at 3 months of age. However, the presence of finger flexion since birth and preserved continuity of the lower trunk implies that the hand function will improve further. Experience has taught us that early restoration of good shoulder and elbow control is important for that recovery.

Hence, the knowledge that intercostal nerve transfers can almost assure return of elbow flexion provides us the latitude to add control of the shoulder, using the available ruptured roots.

The obstacles to harvesting intercostals that are seen following traumatic palsies (fractures at multiple levels with the pleura adherent to the sites of intercostal drains) do not hinder the surgeons here.

The technique of harvesting intercostal nerves differs from that employed in adults. Care has to be taken to avoid stripping the periosteum of the ribs. This proved to be responsible for a deformity of the chest wall with growth in our first patient operated upon more than 20 years ago. Passage through the intercostal muscles, close to the lower border of the rib, allows rapid access to each intercostal nerve. Identification of the nerve anteriorly and following it backward enables us to harvest the four nerves in a short time without excessive loss of blood. The nerves are separated from the pleura by blunt dissection which automatically divides the tiny branches to the intercostal muscles that invariably have to be sacrificed. The authors choose to use four intercostals to ensure an early restoration of a strong biceps, and this has been borne out by the observed results. In addition, approximation to the trunk of the musculocutaneous nerve provides innervation to the biceps and brachialis with stronger elbow flexion. The brachialis can be utilized for distal transfer when required without serious downgrade of elbow flexion.

Unlike in adults, intercostal nerve transfers have proved much more reliable for restoration of strong elbow flexion in babies.

Kawabata ${ }^{3}$ reported on the outcomes in 31 such procedures in 30 patients. They used two intercostals in 26 cases and three in five patients. The mean patient age at surgery was 5.8 months and the mean follow-up period was 5.2 years.

The power of the biceps muscle of the arm was rated Grade M4 in 26 (84\%) of 31 patients.

Luo $^{4}$ reported on 24 patients operated upon at the age of 3 to 11 months and followed-up for 24 to 79 months. The target nerves were musculocutaneous in 12 patients and anterior division of the upper trunk in 12 cases. Four intercostal nerves were transferred in 14 cases, three in nine cases, and two nerves in one case. The nerves were harvested via a zigzag incision at the midaxillary level Grade 3, and stronger function was obtained in 22 of the 24 patients.

Pondaag and Malessy ${ }^{5}$ reported on the use of intercostal nerves and the medial pectoral nerve for restoration of elbow flexion. Till 2000, they used this in all partial palsies with absent roots for grafting (they do not prefer to use fascicles from the ulnar and median nerves) and in all cases of total palsies. The periosteum was spared, and the nerves were harvested through the intercostal muscles, divided as close to the sternum as possible and transferred to the MCN with glue. Three nerves were transferred by direct approximation 
except in one case where a $1 \mathrm{~cm}$ nerve graft had to be interposed because of the dubious quality of the distal stump. Successful biceps reinnervation was obtained in 14 patients (82\%) and three failures occurred.

They had earlier (Pondaag and Malessy) ${ }^{6}$ reported on their outcomes in hand functions in 16 patients afflicted with total palsies. In four patients, intercostal nerve transfers were used for restoration of elbow flexion, and grade 4 function was achieved in each case.

El Gammal ${ }^{7}$ reported on intercostal nerve transfers in 46 patients with birth-related brachial plexus injuries. Of these, 22 patients had total palsies and 19 had extensive partial palsies. They described transfer of three intercostal nerves (3rd to 5 th) to the musculocutaneous nerve in 31 cases, with good and excellent results in 29 of these 31 patients (93.5\%). This is the only report that describes the incidence of atelectasis following surgery which the authors have attributed to general anesthesia, as it was bilateral in one patient. However, only nine patients were evaluated. In our series, we have not noted any respiratory discomfort as a result of the intercostal nerve harvest.

Their 2010 report $^{8}$ described 35 patients afflicted with total birth palsies. Intercostal nerve transfers to the MCN were done in 12 of these patients and independent elbow flexion was successfully achieved in 11 of them (91.6\%).

The results in this series compare favorably with those published in the literature (-Table $\mathbf{1}$ ).

These results are considerably superior to those reported for posttraumatic palsies. As much as $72 \%$ of direct intercostal to musculocutaneous transfers (without interposition nerve grafts) achieved biceps strength M3 or stronger versus $47 \%$ using interposition grafts (Merrell). ${ }^{9}$

The factors mentioned above as well as the young age both contribute to this.

One other indication mentioned in the literature involves the treatment of late presentations or cases primarily operated by nerve grafting in the neck, which develop cocontractions of the biceps and triceps. Kawano ${ }^{10}$ has reported on using intercostals to restore independent elbow flexion in such cases.

In total palsies, the eventual outcome of the upper limb depends entirely on the nerve reconstruction performed at an early stage. Experience has shown that nerve transfers can provide reasonable control of the shoulder and elbow. The available (ruptured roots) have to be judiciously employed for the hand. The quality of wrist and hand functions (closure and opening of the fist, grip strength, and dexterity) depend largely on harnessing the available (ruptured) root stumps to reinnervate the lower trunk. Thus, in the presence of only one or two ruptured roots, it is imperative to divert each of them to the lower trunk. The effect of this strategy is illustrated in a case of total palsy treated with nerve transfers for the suprascapular and MCN and nerve grafting from both ruptured roots to the lower trunk (-Videos $\mathbf{1}$ and $\mathbf{2}$ ). The consistent results of intercostal nerve transfers for restoration of elbow flexion form an integral part of this overall strategy, and allow us to direct the available growing axons from ruptured roots to the shoulder in partial palsies and to the lower trunk for hand functions in total palsies. The hand function achieved in total palsies is the subject of another study that is being prepared for publication.

Videos: Case of total birth palsy left side. Operated upon at 4 months of age. Nerve transfers spinal accessory to suprascapular and intercostals to musculocutaneous nerve and nerve grafting from the ruptured $\mathrm{C} 5$ and $\mathrm{C} 6$ roots to the lower trunk in the neck.

\section{Video 1}

Result at the shoulder elbow showing 45 degrees abduction, almost full external rotation and full elbow flexion and extension. Online content including video sequences viewable at: https://www. thieme-connect.com/products/ ejournals/html/10.1055/s-0040-1716186.

\section{Video 2}

Result at the hand with full flexion of the wrist, fingers and thumb, and full extension of the fingers and thumb. Online content including video sequences viewable at: https://www.thieme-connect.com/products/ejournals/ html/10.1055/s-0040-1716186.

Table 1 The different case series in the literature are listed here

\begin{tabular}{|c|c|c|c|c|c|}
\hline Series & $\begin{array}{l}\text { No. of } \\
\text { cases }\end{array}$ & No. of intercostals & Site of repair & $\begin{array}{l}\text { Follow-up } \\
\text { (years) }\end{array}$ & Success rate \\
\hline Kawabata $^{3}$ & 31 & 2 in 26 cases, 3 in 5 cases & MCN & 5.2 & $91.6 \%$ \\
\hline Pondaag ${ }^{6}$ & 4 & 3 & MCN & 4.2 & $100 \%$ \\
\hline Pondaag $^{5}$ & 17 & 3 & $\mathrm{MCN}$ & 3.9 & $82 \%$ \\
\hline El Gammal $^{7}$ & 31 & 3 & $\mathrm{MCN}$ & 4.1 & $93.5 \%$ \\
\hline El Gammal ${ }^{8}$ & 12 & 3 & MCN & 4.2 & $91.6 \%$ \\
\hline Luo $^{4}$ & 24 & $\begin{array}{l}4 \text { in } 14 \text { cases, } 3 \text { in } 9 \text { cases, } \\
2 \text { in one case }\end{array}$ & $\begin{array}{l}\text { Anterior division of upper trunk in } \\
12 \text { cases, MCN } 12 \text { cases }\end{array}$ & 4.5 & $91.6 \%$ \\
\hline This series & 50 & 4 & MCN & 10 & $96 \%$ \\
\hline
\end{tabular}




\section{Conclusions}

The use of direct transfers of four intercostal nerves to the MCN has proved to be reliable for restoration of independent elbow flexion. This enables us to achieve enhanced overall functional outcomes in total and near-total birth palsies, as the available roots can be utilized better.

\section{Conflicts of Interest}

None declared.

\section{References}

1 Nagano A, Tsuyama N, Ochiai N, Hara T, Takahashi M. Direct nerve crossing with the intercostal nerve to treat avulsion injuries of the brachial plexus. J Hand Surg Am 1989;14(6):980-985

2 Chuang DC, Yeh MC, Wei FC. Intercostal nerve transfer of the musculocutaneous nerve in avulsed brachial plexus injuries: evaluation of 66 patients. J Hand Surg Am 1992;17(5):822-828

3 Kawabata H, Shibata T, Matsui Y, Yasui N. Use of intercostal nerves for neurotization of the musculocutaneous nerve in infants with birth-related brachial plexus palsy. J Neurosurg 2001;94(3):386-391
4 Luo PB, Chen L, Zhou CH, Hu SN, Gu YD. Results of intercostal nerve transfer to the musculocutaneous nerve in brachial plexus birth palsy. J Pediatr Orthop 2011;31(8):884-888

5 Pondaag W, Malessy MJ. Intercostal and pectoral nerve transfers to re-innervate the biceps muscle in obstetric brachial plexus lesions. J Hand Surg Eur Vol 2014;39(6):647-652

6 Pondaag W, Malessy MJ. Recovery of hand function following nerve grafting and transfer in obstetric brachial plexus lesions. J Neurosurg 2006;105(1, Suppl) 33-40

7 El-Gammal TA, Abdel-Latif MM, Kotb MM, et al. Intercostal nerve transfer in infants with obstetric brachial plexus palsy. Microsurgery 2008;28(7):499-504

8 El-Gammal TA, El-Sayed A, Kotb MM, et al. Total obstetric brachial plexus palsy: results and strategy of microsurgical reconstruction. Microsurgery 2010;30(3):169-178

9 Merrell GA, Barrie KA, Katz DL, Wolfe SW. Results of nerve transfer techniques for restoration of shoulder and elbow function in the context of a meta-analysis of the English literature. J Hand Surg Am 2001;26(2):303-314

10 Kawano K, Nagano A, Ochiai N, Kondo T, Mikami Y, Tajiri Y. Restoration of elbow function by intercostal nerve transfer for obstetrical paralysis with co-contraction of the biceps and the triceps. J Hand Surg Eur Vol 2007;32(4):421-426 